\title{
Prayer-Bots and Religious Worship on Twitter: A Call for a Wider Research Agenda
}

\author{
Carl Öhman $^{1}$ (D) Robert Gorwa ${ }^{2}$ [D $\cdot$ Luciano Floridi $^{1,3}$ (D) \\ Published online: 1 April 2019 \\ (c) The Author(s) 2019
}

\begin{abstract}
The automation of online social life is an urgent issue for researchers and the public alike. However, one of the most significant uses of such technologies seems to have gone largely unnoticed by the research community: religion. Focusing on Islamic Prayer Apps, which automatically post prayers from its users' accounts, we show that even one such service is already responsible for millions of tweets daily, constituting a significant portion of Arabic-language Twitter traffic. We argue that the fact that a phenomenon of these proportions has gone unnoticed by researchers reveals an opportunity to broaden the scope of the current research agenda on online automation.
\end{abstract}

Keywords Automatic prayers · Twitter bots · Digital afterlife industry · Islam · Online death

\section{Introduction}

Online social life is increasingly automated. From virtual assistants that help with day-to-day tasks, to chatbots providing companionship or preserving the memory of deceased family members (Öhman and Floridi 2018), industry has been quick in realizing the potential of the development. At the same time, online social automation is also used for political goals, including automated "bot" accounts on social networks that attempt to influence elections and other key political events (Gorwa and Guilbeault 2018). These trends have rightly attracted much attention, both publicly and in the research community. However, one major area of online automation

Carl Öhman

carl.ohman@oii.ox.ac.uk

1 Oxford Internet Institute, University of Oxford, 1 St Giles, Oxford OX1 3JS, UK

2 Department of Politics and International Relations, University of Oxford, Manor Road Building, Manor Road, Oxford OX1 3UQ, UK

3 The Alan Turing Institute, 96 Euston Road, London NW1 2DB, UK 
has largely been overlooked so far: religious worship. In this article, we provide the first large-scale analysis of the religious use of online automation technologies. More specifically, the article focuses on a particularly wide-spread phenomenon, what we call Islamic Prayer Apps, which, despite their popularity, have so far gone unnoticed by the research community. We argue that the spread and social significance of these applications calls for a broadening of the scope of current research on online automation in general, and on social media bots in particular.

\section{Islamic Prayer Apps}

It is increasingly popular amongst Muslim social media users to employ services that automatically post prayers on one's behalf. In this article, we shall refer to such services as Islamic Prayer Apps. These apps vary in their business model and popularity, but share the same goal: to facilitate and automate worship. This does not mean that the apps replace the mandatory "5-times-aday" prayer rituals. While documented services simply send or post reminders for local prayer times (Wyche et al. 2008), the Islamic Prayer Apps seem to facilitate additional public supplication (دُعَاء “dua"), which may be understood as a humble asking for an event to occur or a wish to be fulfilled.

Believers in Islam may phrase their own personal supplications, but there is also an array of examples in the Quran to choose from. Based on these examples, the apps enable the user to post automatically their supplications on social networking sites, like Twitter and Facebook. Du3a.org is a typical example: the site's landing page (see Fig. 1) features some Quranic quotes and popular prayers, and a sidebar encourages visitors to share the site on different social networks, like Facebook and Pinterest, claiming that 26 million visitors have done so already. But the most salient feature is perhaps the button prompting visitors to subscribe to the service. Upon doing so, visitors are redirected to Twitter, where they are asked to authorize the application to use their account and post on their behalf. After a few hours, Du3a begins to post a $>140$ character supplication from the user's account every second hour, alongside a site URL (and until recently a "recycling" emoji).

Because Du3a.org includes the service's URL in every tweet that is sent out from the user's account, its traffic can be measured using Twitter's Streaming application programming interface (API), which provides live access to up to $1 \%$ of Tweets on the global platform. By querying for the dur3a.org URL, we collected tweets posted over a 48-h period, in June 2018. During this time, 3.8 million tweets containing the URL were posted (See Fig. 2). It should be noted however, that Du3a at the time appeared to release one tweet per hour from the users' accounts, a frequency which recently seems to have slowed down to one every second hour. About $50 \%$ of the users self-identify as located in Saudi Arabia and Egypt, suggesting that, at least in the case of Du3a, the phenomenon is predominantly Arabic (other countries represent approximately $1 \%$ each).

The number of 1.9 million tweets per day-coming just from Du3a, one of many Islamic Prayer Apps-demonstrates how much traffic can be generated through 


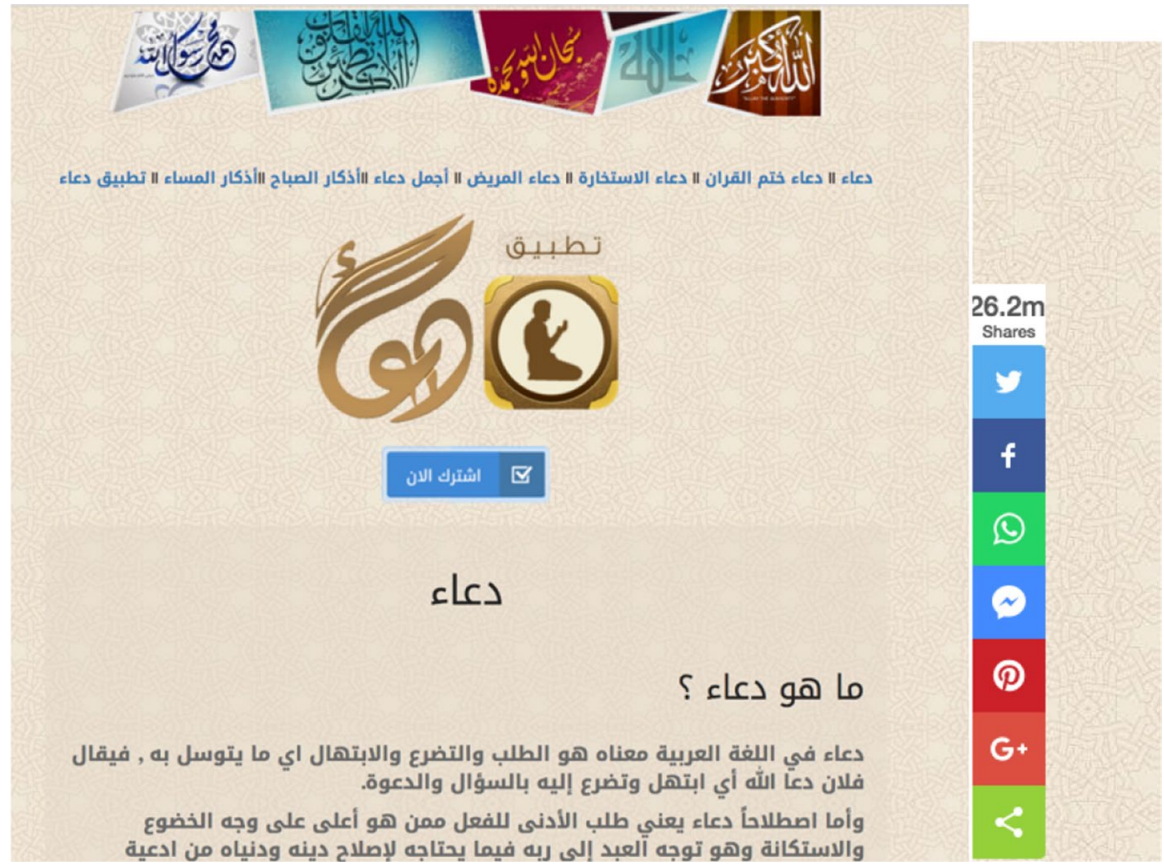

Fig. 1 Screenshot of Du3a's landing page

\section{Tweets Per Day (in Millions)}

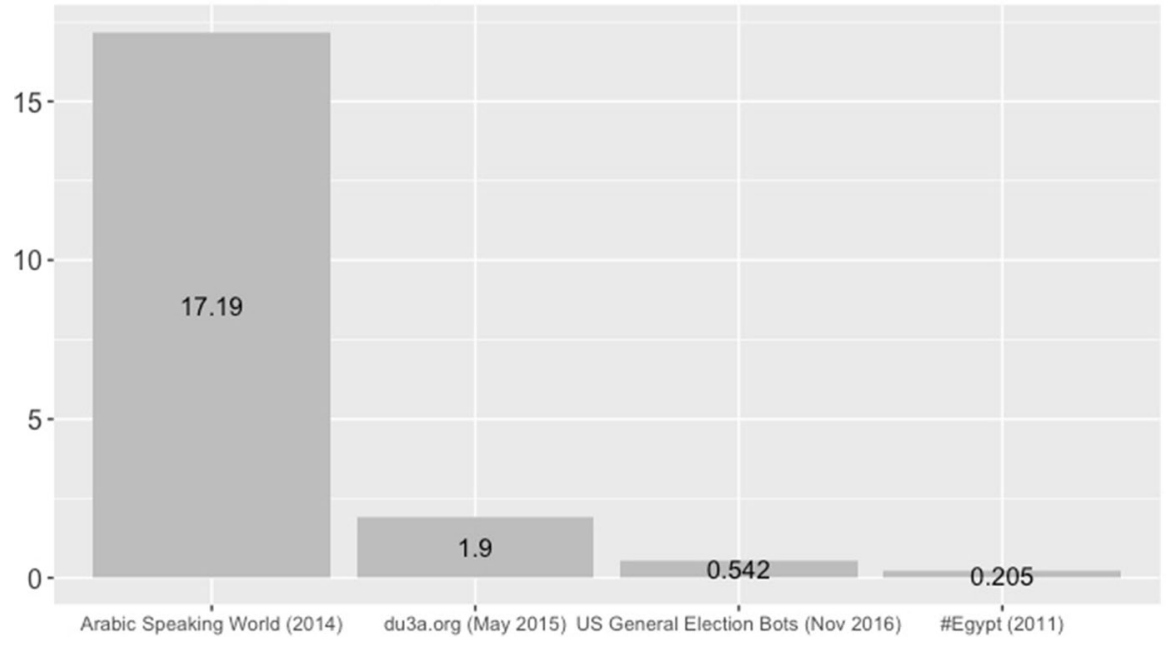

Fig. 2 Illustration of average tweets per day 
automation. To put the numbers in context (see Fig. 2), Bruns et al. (2013) collected 205,000 tweets on the Arab-Spring related hashtag \#egypt on its busiest day, when President Hosni Mubarak resigned amidst intense public pressure. During the 2016 US election, when significant popular attention focused on the role of automated accounts, Bessi and Ferrara (2016) estimated an upper bound of 3.8 million tweets from automated accounts on political topics in the week leading up to voting day (an average of about 540 thousand tweets per day). In other words, according to our exploratory analysis, a single automated prayer app generated almost as many tweets in 2 days as accounts believed to be automated did in the whole week leading up to the US election. Yet, contrary to the US general election, Du3a continues its activity every day of the year. And insofar as we were able to ascertain, this activity has been going on for about 5 years. While exact numbers are difficult to determine, an analysis of Arabic Social Media (2014) estimated that, in 2014, 17.19 million tweets were sent daily from users in the entire Arab world, suggesting that automated prayer may be responsible for a substantial proportion of Twitter in Arabic speaking countries. ${ }^{6}$ Thus, at least in terms of sheer numbers, the expression of worship may rank among the most significant phenomena on Twitter overall.

Du3a.org, like most Islamic Prayer Apps, does not use hashtags which can "trend" and gain visibility, which is a possible reason why the phenomenon has largely remained unnoticed. To our knowledge, it was not until Matthew Rothenberg (2017), the founder of Emojitracker.com, noticed that the recycling emoji (at the time used by Du3a in every tweet) - attributed to the extensive use of the symbol in Muslim tweets - had become the third most popular on Twitter that the apps were first discussed outside the Muslim community.

Our exploration of the phenomenon indicates the presence of at least 10 sites with business models similar to Du3a's. Some of the competitors offer more advanced options. For instance, Athantweets.com offers a premium version that, for 100 Saudi Riyals (roughly \$27) a year, enables the user to choose specific (as opposed to randomly generated) supplications, and for the tweets to be synchronized to the user's local prayer times. Tweets sent via this premium package also hide the Athantweets URL, making them virtually indistinguishable from any other tweets with Quranic content.

This casts light on an important characteristic of the phenomenon as a whole, the fact that a majority of the traffic appears to be organic; that is, derived from ordinary accounts of real people, as opposed to 'bots', understood as accounts with a fake identity set up purely for the purpose of disseminating content. A qualitative close-reading of a few dozen twitter accounts using Du3a shows that, whereas some of the accounts appeared to be created specifically to use these prayer services, most appeared to be ordinary users, who tweeted everyday messages, photos, and commentary interspersed with the automated messages. In other words, much of the traffic appears to be created by authentic accounts, operated by legitimate users, who creatively automate a facet of their online activity while also using the service as they would do ordinarily. 


\section{Religious Context}

It is too soon to try to explain the specific role that the Islamic Prayer Apps play in the everyday life of their users. Much more work on both the qualitative properties of the phenomenon (such as that of Bell 2006) as well as further analysis of the quantitative ones is needed. "Even though there is almost 1.2 billion Muslims ..." as Akma and Abdul Razak (2013, p. 6) point out, “... there is too little research done on the techno-spiritual application from the Islamic perspective." Looking more closely at some of the accounts captured in our data collection, we see that there seem to be many possible motivations behind the use of such services. One account, for instance, notes that the reason it is set up is to pray for "my brother [name]," suggesting that users might be setting up such accounts to cast prayers on behalf of friends and family.

Arguably, one of the core functions of the automated prayer apps is tied to their explicit promise to continue posting even after the user's death. For instance, the slogan of Zad-Muslim.com reads "Register now so your account would tweet now and after you die." Similarly, Dur3a.org promises that "your account will tweet in your life and in your death." This is more than a mere detail. While the posthumous prayer apps resonate with traditions in many different religions, it is notable that such features have emerged within Islam. According to the Quran, one does not receive one's judgement immediately upon death. Instead, those who pass away must wait in their graves for Allah to end Earth and make His final judgment of each respective individual. In order to be eventually sent to paradise, a Muslim's sins must be outweighed by his or her good deeds. But in between the time of death and the final judgement day, a number of factors may posthumously increase their standing in the eyes of Allah. The Prophet Muhammed specifically mentioned three things that can improve one's afterlife reward this way: the continuous effects of charity; the provision of knowledge used by future generations; and virtuous descendants who pray for you (Sahih Muslim 1330, 42: 7064).

Islamic scholars debate about how this should be interpreted (see for instance Al-Halbali 2016). However, most interpreters agree that the reward of the afterlife, at least to some degree, is subject to posthumous improvement. Because contributing to the dissemination of Islam is considered an inherent good, it is less important whether this activity is performed by someone personally, or through knowledge that one helps disseminating. So, the hypothesis is that putting in motion an app to post supplications on one's behalf after one's death could help increase one's chances of a good afterlife.

With few exceptions, the Islamic community has thus far accepted the incorporation of new technologies into religious practice, especially when it comes to realizing their missionary potential. In her book When Religion Meets New Media, Campbell (2010, p. 96) describes this new emphasis on technology, noting that "Engagement with media is a religious imperative, one which Muslims will be held accountable for in the afterlife." Thus, it seems that the dissemination of Islam through media may have profound implications in both life and death. Yet, little is known about the attitudes of the larger Islamic community 
when it comes to Islamic Prayer Apps, which undoubtedly ties religious practice closer to online technologies. And research is needed to study the specific role that these apps may play within the lives and religious practices of their users. Likewise, little, if anything, is known about the stance of social media platforms themselves on this widespread phenomenon.

Unlike a platform such as Facebook, which now has a "memorialization" feature, Twitter handles deceased users by permanently taking down their accounts after some months of inactivity (Twitter n.d.). However, an account that has subscribed to a Islamic Prayer App will not go inactive after the user's death. It will keep tweeting, and will therefore not be identified as inactive or abandoned. This means that the huge presence of the Islamic Twitter supplications will likely continue to grow long after the account holders have died. An array of similar applications, albeit with a secular framing of "immortalizing one's social media presence", have been launched mainly targeting secular Western audiences (Öhman and Floridi 2017). However, such a project of social media immortalization still remains fringe in comparison to the Islamic Prayer Apps. Considering their popularity, it may, even within only a few decades, become increasingly difficult to differentiate between traffic generated by living and deceased users - and not because of the futurist community in Silicon Valley, but because of Islamic worshipers.

\section{Broader Implications}

Religion has always been one of the most significant aspects of human life, individually, socially, as well as technologically. It should not be surprising if it is now emerging as a possible key driver for the mainstream adoption of social technology. Islam is not the only religion incorporating creative automation technologies into worship. The iTunes App Store contains more than 6000 applications related to spirituality and religion (Buie and Blythe 2013, p. 2315). In early 2019, Pope Francis launched a new app called "Click to Pray,"1 with which Catholics may participate in the Pope's prayers and share them on social media. Similarly, the Church of England's new voice activation feature for Amazon's Alexa allows owners of the device to ask it to read daily prayers (BBC 2018). This could be understood as part of a larger movement; after all, the offering of a wax candle in a church can now often be replaced by the turning on of an electric one. In a narrow capacity, technological services can even substitute for priests, answering religious questions like "Who is God?" or what it means to believe in Jesus. Similarly, Jewish communities have started employing new technologies to automate home facilities during the Sabbath (Woodruf et al. 2007), and members of diverse religious communities can now chat and make prayer requests to religious chatbots, such as those created by the Californian start-up Prayerbot. These examples, alongside the scale of the Islamic Prayer Apps, show that religion is far from a small or marginal force in contemporary social automation.

\footnotetext{
${ }^{1}$ https://clicktopray.org/.
} 
The case of the Islamic Prayer App also provides valuable insights into the complex, fast-evolving discourse on Twitter bots and online automation policy. Much recent literature on social bots focuses on false accounts set up during elections, and other political events, to inflate engagement metrics and help spread problematic political content (Ferrara et al. 2016). In contrast, Islamic Prayer Apps share no hashtags, and do not appear to try and influence the social network, instead remaining unobtrusively out of view. As well, because many of the observed accounts appear to be ordinary users who have partially "botified" or automated their accounts, they complicate the existing discourse, which is often methodologically and conceptually predicated on the assumption that "bots" and "not bots" exist as two distinct categories that can be easily separated (Gorwa and Guilbeault 2018; Stieglitz et al. 2017). Many Twitter users rely on a variety of publicly available services, from Twitter's own platform 'Tweetdeck' to 'If This Then That' to automate parts of their online activity. But how exactly should such behavior be understood? And what are the ethical ramifications?

There have been many positive uses of automated social media accounts, which have been deployed creatively by journalists (Lokot and Diakopoulos 2016), activists fighting corruption (Savage et al. 2016), and those promoting institutional transparency (Ford et al. 2016), but religious uses have been largely unexplored. The Islamic Prayer Apps we analysed here arguably represent one of the largest examples to date of Twitter automation being deployed organically in a creative and culturally significant way. The fact that a phenomenon of these unprecedented proportions has gone unnoticed by researchers shows the limitations of our current scope. To quote Bell (2006, p. 155): "We appear to be stubbornly secular in our imaginings of home and leisure contexts of computing." Indeed, now is the time to broaden the conversation.

Acknowledgements Our sincere thanks to Bence Kollanyi, for assistance with data collection.

Open Access This article is distributed under the terms of the Creative Commons Attribution 4.0 International License (http://creativecommons.org/licenses/by/4.0/), which permits unrestricted use, distribution, and reproduction in any medium, provided you give appropriate credit to the original author(s) and the source, provide a link to the Creative Commons license, and indicate if changes were made.

\section{References}

Akma, N., \& Abdul Razak, F. H. (2013). On the emergence of techno-spiritual: The concepts and current issues. In Computer and mathematical sciences graduates national colloquium 2013 (SISKOM2013).

Al-Halbali, I. J. (2016). The three that follow to the grave. Birmingham: Dar As-Sunnah Publishers.

Arab Social Media Report. (2014). Twitter in the Arab Region. Available at: http://arabsocialmediarepor t.com/Twitter/LineChart.aspx. Accessed 12 June 2018.

BBC. (2018). Church of England offers prayers read by Amazon's Alexa. BBC.com. https://www.bbc. co.uk/news/uk-44233053. Accessed 27 Mar 2019.

Bell, G. (2006). No more SMS from Jesus: Ubicomp, religion and techno-spiritual practices. In P. Dourish \& A. Friday (Eds.), UbiComp 2006: Ubiquitous Computing. UbiComp 2006. Lecture Notes in Computer Science, vol 4206. Springer, Berlin, Heidelberg. https://doi.org/10.1007/11853565_9. 
Bessi, A., \& Ferrara, E. (2016). Social bots distort the 2016 US Presidential election online discussion. First Monday. https://doi.org/10.5210/fm.v21i11.7090.

Bruns, A., Highfield, T., Burgess, J. (2013). The Arab spring and social media audiences. American Behavioral Scientist, 57(7):871-898.

Buie, E., \& Blythe, M. (2013). Spirituality: There's an App for That! (But not a lot of research). CHI 2013 Extended Abstracts, April 27-May 2, 2013, Paris, France.

Campbell, H. A. (2010). When religion meets new media. London: Routledge.

Ferrara, E., Varol, O., Davis, C. B., Menczer, F., \& Flammini, A. (2016). The rise of social bots. Coтmunications of the ACM, 59(7), 96-104.

Ford, H., Dubois, E., \& Puschmann, C. (2016). Keeping Ottawa honest-One tweet at a time? Politicians, journalists, wikipedians and their twitter bots. International Journal of Communication, 10, 4891-4914. ISSN 1932-8036.

Gorwa, R., \& Guilbeault, D. (2018). Unpacking the social media bot: A typology to guide research and policy. Policy and Internet. https://doi.org/10.1002/poi3.184.

Lokot, T., \& Diakopoulos, N. (2016). News Bots: Automating news and information dissemination on Twitter. Digital Journalism, 4(6), 682-699. https://doi.org/10.1080/21670811.2015.1081822.

Öhman, C., \& Floridi, L. (2017). The political economy of death in the age of information: A critical approach to the digital afterlife industry. Minds and Machines. https://doi.org/10.1007/s1102 3-017-9445-2.

Öhman, C., \& Floridi, L. (2018). An ethical framework for the digital afterlife industry. Nature Human Behavior. https://doi.org/10.1038/s41562-018-0335-2.

Rothenberg, M. (2017). Why the emoji recycling symbol is taking over Twitter. Medium. Available at: https://medium.com/@ mroth/why-the-emoji-recycling-symbol-is-taking-over-twitter-65ad4b18b0 4b. Accessed 25 Apr 2018.

Sahih Muslim. (1330). Sahih Muslim Vol. 7, Book of Zuhd and Softening of Hearts, Hadith 7064. Retrieved from: http://www.iupui.edu/ msaiupui/042.smt.html. Accessed 27 Mar 2019.

Savage, S., Monroy-Hernandez, A., Höllerer, T. (2016). Botivist: Calling volunteers to action using online bots. In Proceedings of the 19th ACM Conference on Computer-Supported Cooperative Work \& Social Computing, pp. 813-822. ACM.

Stieglitz, S., Brachten, F., Ross, B., \& Jung, A.-K. (2017). Do social bots dream of electric sheep? A categorisation of social media bot accounts. arXiv:1710.04044 [Cs]. Retrieved from http://arxiv.org/ abs/1710.04044.

Twitter. (n.d.). Inactive account policy. Available at: https://help.twitter.com/en/rules-and-policies/inact ive-twitter-accounts. Accessed 27 Mar 2019

Woodruf, A., Augustin, S., \& Foucault, B. (2007). Sabbath day home automation: "It's like mixing technology and religion". CHI 2007, April 28-May 3, 2007, San Jose, California, USA.

Wyche, S. P., Caine, K. E., Davison, B., Arteaga, M., \& Grinter, R. E. (2008). Sun dial: Exploring technospiritual design through a mobile islamic call to prayer application. CHI 2008, April 5 - April 10, 2008, Florence, Italy ACM.

Publisher's Note Springer Nature remains neutral with regard to jurisdictional claims in published maps and institutional affiliations. 